\title{
Explanation-based learning with weak domain theories
}

\author{
Michael J. Pazzani (pazzani@ics.uci.edu) \\ Department of Information and Computer Science \\ University of California \\ Irvine, CA 92717
}

Much knowledge about the world does not consist of universal generalizations. Instead, it consists of less certain generalizations of tendencies that occur in absence of other factors. Cognitive psychology research has shown that prior background knowledge influences the rate or accuracy of learning. However, computational models of learning have assumed that this background knowledge consists of universal generalizations. Here, I relax this assumption by considering background knowledge consisting of tendencies or influences. The following quote illustrates a more realistic type of background knowledge [1]:

Straightforward theory relates injuries from falls to three sets of variables... First, the height of the fall determines the impact velocity. Second, the softness of the surface of impact affects the stopping distance and hence the impact force.... And third, at least five properties of the falling body itself are relevant: its mass, which determines the impact force $(F)$; its cross-sectional area $A$, determining the frictional drag during the fall and also the stress upon impact (F/A); cross-sectional areas of bone, determining bone strength; cushioning of vital body parts by fat and other soft tissue; and dissipation of impact forces through flexing of muscles and use of joints... because mass increases as the cube, but surface area as the square of linear dimensions, falling large animals are in general more injury prone than small ones, as they suffer greater impact stress, and they reach higher terminal velocities in freefall because of the less favorable arealmass ratio.

One facet of a weak domain theory is that the influence of a number of factors is known. A weak theory does not provide any means of combining these influences. Will an elephant survive a 10 foot fall into a snow bank? Will a mouse survive a 5 foot fall onto concrete if it lands on its head? These are empirical questions. However, the theory does constrain the features which play a part in predictive relationships. Only when an accurate predictive relationship cannot be made by considering combinations of known influences are other factors considered.

We have constructed a learning system called POSTHOc that uses this sort of background knowledge to propose hypotheses that are then tested against further data. Post Hoc utilizes a weak domain theory to generate plausible explanations for a state change after it has occurred. This background knowledge is also used to revise hypotheses that fail to make accurate predictions.

POSTHOC has been tested on a problem of predicting when a balloon will be successfully inflated. Given this weaker form of background knowledge, it is possible to produce several explanations for why a particular outcome occurred. Since different explanations make different predictions about other cases, additional examples are needed to rule out alternatives. Since the set of hypothesis which are consistent with the background knowledge and the data is much smaller than the set of hypothesis that are consistent with only the data, fewer examples are required to learn to make accurate predictions. The following two influences are used as background knowledge:

(easier (strong-actor) (inflate balloon))

(easiex (less-elastic) (inflate balloon))

Given this background knowledge it is not clear whether both conditions are necessary or if either is sufficient. Thus given this weaker form of background knowledge, it is possible to produce several explanations for why a particular outcome occurred. To determine when these influences are present in a given example POSTHOC has a set of inference rules. The following rules represent the facts that stretching a balloon tends to make it less elastic; that older actors tend to be stronger actor; and that adults are old:

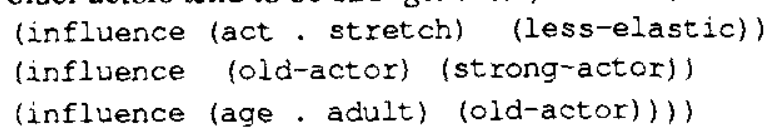


POSTHOC maintains a single hypothesis that consists of a disjunction of conjunctions. For example, the following represents the hypothesis that adults can inflate any balloon, or children can inflate yellow balloons:

((lage. adult)) ((age. child) (color. yellow)) ) $>$ (inflate balloon)

When the current hypothesis makes an error, a set of rules examines the hypothesis and the incorrectly classified example, and revises the hypothesis. There are three sets of rules. One set deals with errors of commission in which a positive example is falsely classified as a negative example. This rule set makes the hypothesis more general. Another rule set deals with errors of omission. This rule set makes the hypothesis more specific. The final rule set creates an initial hypothesis when the first positive example is encountered. Within each rule set, the rules are ordered by priority. In this paper, we present the subset of the rules that are necessary to follow the example.

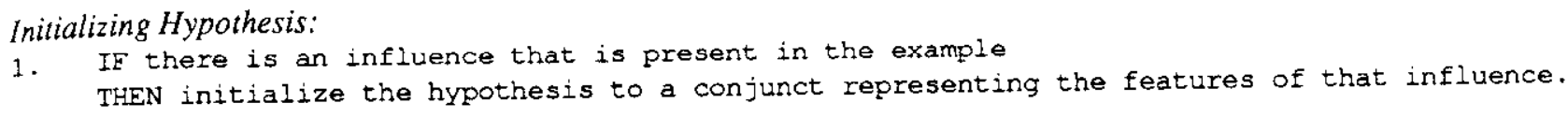

This rule determines if there are features of the example that would influence the outcome of a positive example by chaining backward from the rules that indicate that a certain outcome is easier under certain conditions. The conditions are verified by chaining backward via influence rules to find features that are indicative of an influence.

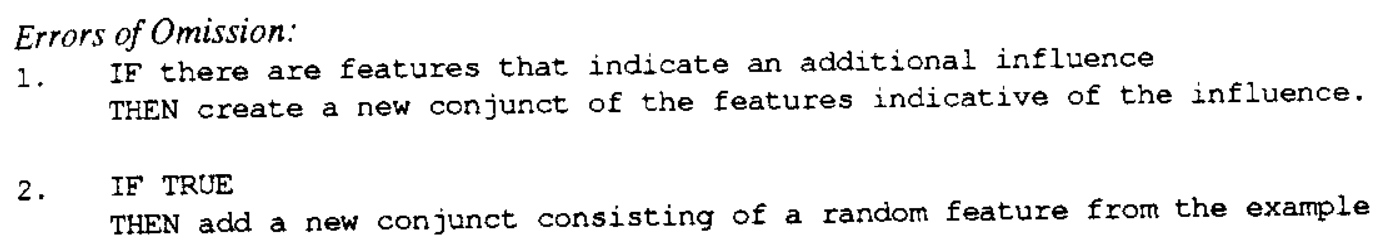

The first rule applies if the features of the example indicate the presence of an additional factor. This additional factor is assumed to be a multiple sufficient cause (cf. [2]) and a new conjunct is added to specialize the hypothesis. The second rule forms an additional conjunct from a random feature of the example when hypotheses consistent with background knowledge have been ruled out. This random choice is needed because we do not explore multiple consistent hypotheses in parallel and the background knowledge could not guide the selection of a feature.

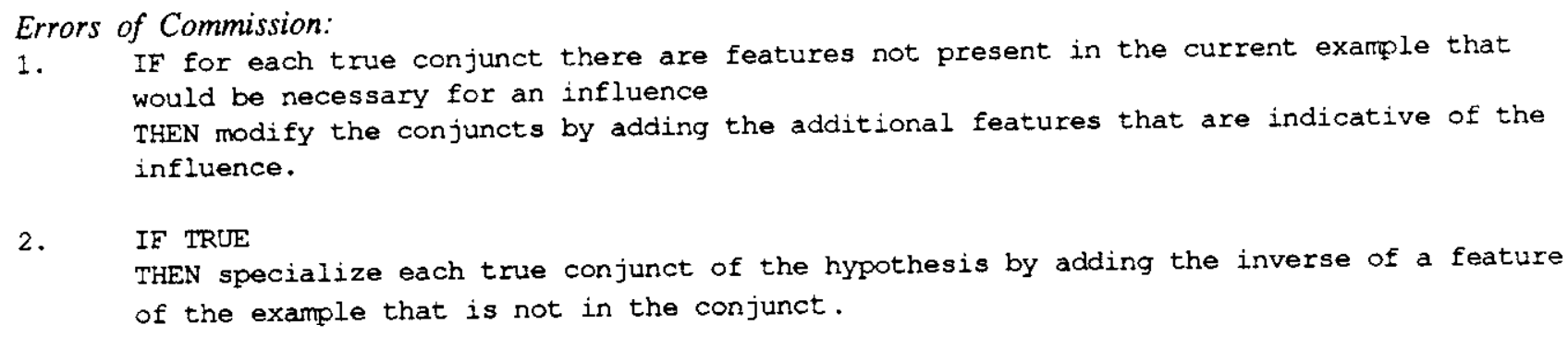

The first rule adds a multiple necessary cause to the hypothesis [2]. For example, if the hypothesis is that all adults can inflate balloons, an error will occur on an example of an adult not inflating a large yellow balloon that has been dipped in water. The hypothesis is modified by finding an additional factor which could affect the outcome that is not present in the example (stretching the balloon) and asserting that this is necessary to inflate the balloon. The new hypothesis consists of a single conjunct that represents the prediction that adults can only inflate balloons that have been stretched. The second rule specializes a hypothesis by adding additional features to each true conjunct.

An example of POSTHOC acquiring a predictive rule will help to clarify how hypotheses are formed and revised. Here, we consider how POSTHOC operates with an incomplete theory with only one influence present:

(easier (less-elastic) (inflate balloon)).

This example will illustrate how both the analytical and empirical components cooperate to create a hypothesis. The first example is an example of an adult inflating a yellow balloon that had been stretched. Rule 1 from the 
initialization rule set finds an influence present and the initial hypothesis is that all balloons that have been stretched can be inflated:

$((($ act . stretch $))) \Rightarrow($ inflate balloon $)$

This hypothesis is consistent with several more examples. Finally, an error of omission occurs when POSTHOC predicts that a balloon will not be inflated, but it is:

((color. red) (size. large) (act. dip) (age . adult)) $\Rightarrow$ (inflate balloon)

The second rule in the error of omission set randomly selects one feature and makes a new conjunct. This feature is dipping the balloon in water. The new hypothesis states that stretching a balloon or dipping a balloon in water are predictive of the balloon being inflated:

$((($ act . stretch $))(($ act $\cdot \operatorname{dip}))) \Rightarrow$ (inflate balloon)

This hypothesis causes an error of commission when an example is erroneously predicted to result in a successful inflation of a balloon:

((color. red) (size. small) (act. dip) (age. child)) $>$ (not (inflate balloon))

The second rule for errors of commission specializes the conjunct that indicates that dipping a balloon in water is predictive of the balloon being inflated. The inverse of the age is selected as an additional necessary condition for this conjunct. The new hypothesis indicates that an adult can inflate a balloon that has been dipped in water, and anyone can inflate a balloon that had been stretched. This hypothesis is consistent with the remaining data.

(( (age . adult) (act. dip)) ((act. stretch))) $\Rightarrow$ (inflate balloon)

The previous example illustrated a fortuitous ordering of examples and some lucky random choices. We have tested Post Hoc with varying amounts of background knowledge on randomly ordered examples. When Post Hoc is run with no domain theory, it takes longer to converge on an accurate concept definition than with a partial theory. When POSTHOC is run with a complete (but weak) domain theory, it converges more rapidly. Figure 1 shows the results of running POSTHoC (averaged over 200 trails) under these three conditions to acquire the previous rule.

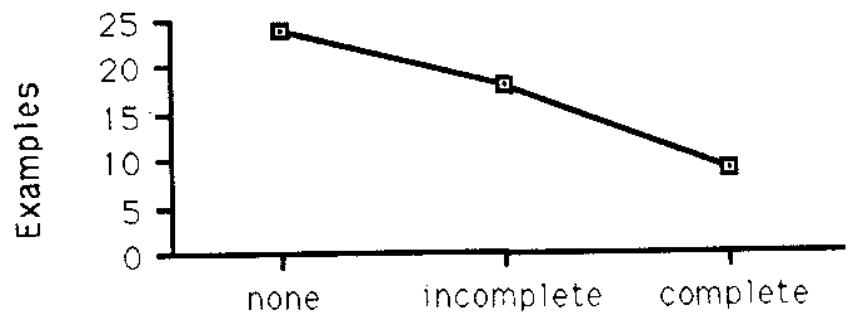

Figure 1. The number of examples required to converge on an accurate hypothesis.

This model extends analytical learning techniques to problems that do not have a domain theory sufficiently strong to deductively predict outcomes. With this weaker form of domain theory, hypotheses must be tested and revised to reflect observed regularities. In the future, we plan to extend this model to deal with negative influences and more complex interactions between influences. In addition, we plan to extend the domain theory to account for new influences present in the data. Finally, we intend to investigate techniques for dealing wilh probabilistic relationships.

\section{Acknowledgements}

This work was funded in part by a Faculty Research Grant from the University of California, Irvine.

\section{References}

[1] Diamond, J. (1988) Why cats have nine lives. Nature Vol 332, 586-587 (14 April).

[2] Kelley, Harold H. (1971). Causal Schemata and the Attribution Process. In Jones, Edward E., Kanouse, David E., Kelley, Harold H., Nisbett, Richard E., Valins, Stuart \& Weiner, Bernard (Ed.), Attribution: Perceiving the Causes of Behavior. Morristown, NJ: General Learning Press. 